\title{
Multichannel effects in Rashba quantum wires
}

\author{
M. M. Gelabert, ${ }^{1}$ Llorenç Serra, ${ }^{1,2}$ David Sánchez, ${ }^{1,2}$ and Rosa López ${ }^{1,2}$ \\ ${ }^{1}$ Departament de Física, Universitat de les Illes Balears, E-07122 Palma de Mallorca, Spain \\ ${ }^{2}$ Institut de Física Interdisciplinar i de Sistemes Complexos (IFISC), CSIC-UIB, E-07122 Palma de Mallorca, Spain
}

(Received 29 January 2010; published 26 April 2010)

\begin{abstract}
We investigate intersubband mixing effects in multichannel quantum wires in the presence of Rashba spin-orbit coupling and attached to two terminals. When the contacts are ferromagnetic and their magnetization direction is perpendicular to the Rashba field, the spin-transistor current is expected to depend in an oscillatory way on the Rashba coupling strength due to spin coherent oscillations of the traveling electrons. Nevertheless, we find that the presence of many propagating modes strongly influences the spin precession effect, leading to (i) a quenching of the oscillations and (ii) strongly irregular curves for high values of the Rashba coupling. We also observe that in the case of leads' magnetization parallel to the Rashba field, the conductance departs from a uniform value as the Rashba strength increases. We also discuss the Rashba interaction-induced currentpolarization effects when the contacts are not magnetic and investigate how this mechanism is affected by the presence of several propagating channels.
\end{abstract}

DOI: 10.1103/PhysRevB.81.165317

PACS number(s): 71.70.Ej, 72.25.Dc, 73.63.Nm

\section{INTRODUCTION}

Since the discovery of the giant magnetoresistance effect, ${ }^{1,2}$ research in spintronics has been developing at a fast pace. An important requirement for practical applications of this novel technology is the generation, control, and manipulation of spin-polarized currents preferably using electric fields only. ${ }^{3}$ Spin-orbit interactions in semiconductor materials are promising tools to achieve that goal. In particular, the Rashba interaction, ${ }^{4}$ a type of spin-orbit coupling that originates from a lack of inversion symmetry in semiconductor heterostructures (such as InAs or GaAs), has been experimentally shown to possess a high degree of tunability using gate contacts. ${ }^{5,6}$

Since the spin-orbit interaction couples the electron momentum and its spin, the Rashba field behaves as an effective magnetic field that is responsible for spin coherent oscillations, which can be exploited in spintronics. Based on this property, Datta and Das ${ }^{7}$ suggested a spin field-effect transistor. It consists of a one-dimensional (1D) ballistic channel sandwiched by two ferromagnetic contacts. Their proposal relies on the control of the current along the channel using the Rashba interaction via a third terminal (the gate) and the relative orientation of the leads' magnetizations. The length of the channel and the intensity of the Rashba strength determine the flow of the current. Realization of the spin transistor was hindered by some limitations, such as the mismatch problem (which results in poor injection of spinpolarized current between a ferromagnet and a semiconductor $)^{8}$ and the idealization of ballistic transport. ${ }^{9}$ However, recent experiments on quasi-two-dimensional (2D) structures have overcome these obstacles and have obtained a behavior which looks similar to the spin-transistor effect. ${ }^{10}$

In reality, strictly one-dimensional channels are hard to fabricate and one must deal mostly with quasi-onedimensional systems containing many propagating channels. Confinement in the transversal direction is accomplished with potentials leading to subband spacings often smaller than a few meV, the order of magnitude of the Fermi energy in low-dimensional systems. As a consequence, multiple subbands are populated and channel mixing effects become relevant in many situations. In fact, the Rashba interaction itself includes an intersubband mixing term which couples adjacent subbands with opposite spins. This coupling has been recently demonstrated to give rise to strongly modulated conductance curves, ${ }^{11-14}$ especially close to the onset of higher-energy plateaus, due to Fano interference ${ }^{15}$ between propagating waves and Rashba induced localized levels. ${ }^{13}$ In the presence of in-plane magnetic fields, Rashba couplinginduced intersubband mixing effects are shown ${ }^{16}$ to reduce the visibility of anomalous conductance steps ${ }^{17}$ and to produce transmission asymmetric line shapes even in purely one-dimensional systems. ${ }^{18}$ The opposite $2 \mathrm{D}$ limit of vanishing transverse confinement was studied for spintronic transport by holes in Ref. 19, confirming for this case the feasibility of the spin-transistor effect, and, more recently, in Refs. 20 and 21 to analyze the experiments by Koo et al. ${ }^{10}$

In this paper, we analyze the role of intersubband coupling effects in multichannel quantum wires. Our model consists of a quantum wire with a localized Rashba spin-orbit interaction coupled to ferromagnetic leads with magnetization perpendicular to the direction of the Rashba field. We find that the Rashba intersubband coupling term modifies the spin precession effect in a dramatic way. Typically, one finds a few oscillation cycles in the conductance curves before arriving at a strongly irregular domain at high values of the Rashba parameter in which case the intersubband coupling produces an effective randomization of the injected spins independent of the relative orientation of the leads' magnetization. Therefore, our results point out a serious limitation of the spin-transistor performance, even in the ideal cases of perfect spin injection and fully ballistic propagation.

On the other hand, Rashba interaction has lately deserved much attention as a generation procedure of spin-polarized currents. Several methods have been proposed in different setups (see Refs. 22-43, although the list is by no means exhaustive). We here consider a simple system: a Rashba quantum wire attached to two nonmagnetic leads. We find that the Rashba interaction can produce a highly polarized 
a)

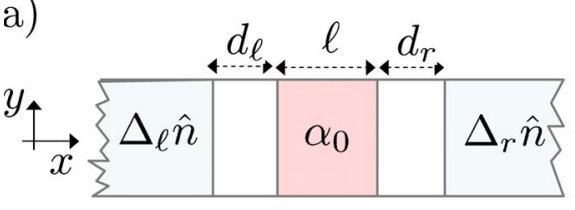

b)

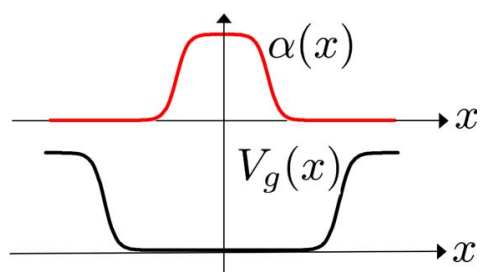

$$
\mathcal{H}=-\frac{\hbar^{2}}{2 m}\left(\frac{d^{2}}{d x^{2}}+\frac{d^{2}}{d y^{2}}\right)+\frac{1}{2} m \omega_{0}^{2} y^{2}+V_{g}(x)+\Delta(x) \hat{n} \cdot \vec{\sigma}+\mathcal{H}_{R}
$$

The confinement along the direction $y$, perpendicular to the current, is taken as parabolic with oscillator frequency $\omega_{0}$, which defines the length $\ell_{0}=\sqrt{\hbar / m \omega_{0}}$. The inhomogeneous Rashba coupling $\mathcal{H}_{R}$ is given by

$$
\mathcal{H}_{R} \equiv \mathcal{H}_{R}^{(1)}+\mathcal{H}_{R}^{(2)}=\frac{\alpha(x)}{\hbar} p_{y} \sigma_{x}+\left[-\frac{\alpha(x)}{\hbar} p_{x}+\frac{i}{2} \alpha^{\prime}(x)\right] \sigma_{y},
$$

FIG. 1. (Color online) Sketch of the physical system (a) and of the spatial variation of Rashba intensity $\alpha(x)$ and gate potential $V_{g}(x)(\mathrm{b})$.

electric current and that the effect is purely due to interchannel coupling. For quantum waveguides supporting a single propagating mode, the polarization effect vanishes. ${ }^{44-46}$ Since the Rashba interaction is localized, we calculate the generated polarization as a function of the interface smoothness and show that the highest values of the polarization are obtained when the transition between the regions with and without spin-orbit interaction is abrupt.

In Sec. II we discuss the physical system and establish the theoretical model to calculate the linear conductance. Section III is devoted to the numerical results when the contacts are ferromagnetic. The spin polarization effect in the case of normal contacts is analyzed in Sec. IV. Finally, Sec. V contains our conclusions.

\section{PHYSICAL SYSTEM AND MODEL}

We consider a quasi-one-dimensional system (a quantum wire) with a localized Rashba interaction (the Rashba dot) coupled to semi-infinite leads. Figure 1 shows a sketch of the physical system. Transport occurs along the $x$ direction. We characterize the Rashba dot as a small region of length $\ell$ with strong spin-orbit coupling with strength $\alpha_{0}$. The spin polarization in the leads is described using the Stoner model for itinerant ferromagnets. Due to exchange interaction among the electrons, the electronic bands in the asymptotic regions become spin split with a splitting phenomenologically given by an effective field $\Delta_{0}$, which we take as a parameter. This approximation is good at low temperatures (lower than the Curie temperature) and for electron densities large enough so that strong correlations can be safely neglected. ${ }^{47}$ Denoting the Stoner field in left and right regions by $\Delta_{\ell}$ and $\Delta_{r}$, respectively, the parallel configuration is described by $\Delta_{\ell}=\Delta_{r}=\Delta_{0}$ while the antiparallel corresponds to $\Delta_{\ell}=-\Delta_{r}=\Delta_{0}$. In addition, we assume that a local gate potential $V_{g}(x)$ is aligning the potential bottom of the successive regions. This way we remove unwanted conductance modifications due to the potential mismatches, ${ }^{8}$ thus focusing on the properties induced purely by the spin-orbit coupling.

The system Hamiltonian reads where, as usual, spin is represented by the vector of Pauli matrices $\vec{\sigma}$ while $p_{x}$ and $p_{y}$ are the Cartesian components of the electron's linear momentum. The Rashba intensity $\alpha(x)$ varies smoothly taking a constant value $\alpha_{0}$ inside the Rashba dot and vanishing elsewhere. The term proportional to $p_{x}$ is responsible for spin precession of an injected electron. ${ }^{7}$ The intersubband coupling term proportional to $p_{y}$ couples adjacent subbands with opposite spins. Finally, the term with the derivative $\alpha^{\prime}(x)$ is added in Eq. (2) to ensure the Hermitian character of the Hamiltonian with the usual symmetrized operator generalization: $\alpha_{0} p_{x} \rightarrow\left[\alpha(x) p_{x}+p_{x} \alpha(x)\right] / 2$.

As mentioned above, the Stoner field $\Delta(x)$ is constant in the left and right asymptotic regions $\left(\Delta_{\ell, r}\right)$ and it smoothly vanishes at distances $d_{\ell, r}$ toward the left and right of the Rashba dot. These are assumed large enough such that all evanescent states at the interface vanish before reaching the leads. The gate potential aligning the band bottom of the different regions is taken as $V_{g}(x)=|\Delta(x)|$. An equivalent choice but localized to the Rashba dot would be $V_{g}(x)$ $=|\Delta(x)|-\Delta_{0}$. All spatial transitions in $\alpha(x)$ and $\Delta(x)$ are described using Fermi-type functions characterized by a small diffusivity $a{ }^{49}$ In general, $a$ is assumed to be small enough although we shall also discuss below the dependence with this parameter in some cases.

For a given energy $E$ the electron wave function fulfills Schrödinger's equation

$$
(\mathcal{H}-E) \Psi=0
$$

with the appropriate boundary conditions. Our method of solution combines discretization of the longitudinal variable $x$ in a uniform grid with a basis expansion in transverse eigenfunctions $\phi_{n}(y)$ and in eigenspinors $\chi_{s}(\eta)$ along a direction given by a unitary vector $\hat{n}$

$$
\Psi=\sum_{s= \pm} \sum_{n=0}^{\infty} \psi_{n s}(x) \phi_{n}(y) \chi_{s}(\eta),
$$

where $s= \pm$ is the spin quantum number while $\eta=\uparrow, \downarrow$ denotes the twofold spin discrete variable. In terms of the polar and azimuthal angles $(\theta, \phi)$ corresponding to the spin quantization axis $\hat{n}$ we can write 


$$
\chi_{+} \equiv\left(\begin{array}{c}
\cos \left(\frac{\theta}{2}\right) \\
\sin \left(\frac{\theta}{2}\right) e^{i \phi}
\end{array}\right) ; \chi_{-} \equiv\left(\begin{array}{c}
\sin \left(\frac{\theta}{2}\right) \\
-\cos \left(\frac{\theta}{2}\right) e^{i \phi}
\end{array}\right)
$$

The transverse eigenfunctions are the solutions of the harmonic 1D oscillator

$$
\left(-\frac{\hbar^{2}}{2 m} \frac{d^{2}}{d y^{2}}+\frac{1}{2} m \omega_{0}^{2} y^{2}\right) \phi_{n}(y)=\varepsilon_{n} \phi_{n}(y)
$$

with

$$
\varepsilon_{n}=\left(n+\frac{1}{2}\right) \hbar \omega_{0} ; \quad n=0,1, \ldots
$$

Projecting Eq. (3) onto the basis we obtain the equations for the unknown channel amplitudes $\psi_{n s}(x)$

$$
\begin{gathered}
-\frac{\hbar^{2}}{2 m} \psi_{n s}^{\prime \prime}(x)+\left[V_{g}(x)+s \Delta(x)+\varepsilon_{n}-E\right] \psi_{n s}(x) \\
+\sum_{n^{\prime} s^{\prime}}\left\langle n s\left|\mathcal{H}_{R}\right| n^{\prime} s^{\prime}\right\rangle \psi_{n^{\prime} s^{\prime}}(x)=0 .
\end{gathered}
$$

Notice that the Rashba interaction is the only source of interchannel coupling since, in general, the matrix element $\left\langle n s\left|\mathcal{H}_{R}\right| n^{\prime} s^{\prime}\right\rangle$ will be nondiagonal. Using the separation in two spin-orbit contributions introduced in Eq. (2) we can write

$$
\begin{gathered}
\left\langle n s\left|\mathcal{H}_{R}^{(1)}\right| n^{\prime} s^{\prime}\right\rangle=\frac{\alpha(x)}{\hbar}\left\langle n\left|p_{y}\right| n^{\prime}\right\rangle\left\langle s\left|\sigma_{x}\right| s^{\prime}\right\rangle, \\
\left\langle n s\left|\mathcal{H}_{R}^{(2)}\right| n^{\prime} s^{\prime}\right\rangle=\left[-\frac{\alpha(x)}{\hbar} p_{x}+\frac{i}{2} \alpha^{\prime}(x)\right] \delta_{n n^{\prime}}\left\langle s\left|\sigma_{y}\right| s^{\prime}\right\rangle .
\end{gathered}
$$

Equations (9) and (10) clearly show that, in general, both $\mathcal{H}_{R}^{(1)}$ and $\mathcal{H}_{R}^{(2)}$ couple channels with opposite spins through the matrix elements $\left\langle s\left|\sigma_{x}\right| s^{\prime}\right\rangle$ and $\left\langle s\left|\sigma_{y}\right| s^{\prime}\right\rangle$. Of course, if the spin quantization axis $\hat{n}$ is chosen along the $x$ or $y$ axis then either $\left\langle s\left|\sigma_{x}\right| s^{\prime}\right\rangle$ or $\left\langle s\left|\sigma_{y}\right| s^{\prime}\right\rangle$ become diagonal. Regarding the coupling between transverse modes, we notice that $\mathcal{H}_{R}^{(2)}$ is always diagonal $\left(\delta_{n n^{\prime}}\right)$ while $\mathcal{H}_{R}^{(1)}$ is connecting modes differing in one subband index $\left(n^{\prime}=n \pm 1\right)$ through the oscillator matrix element $\left\langle n\left|p_{y}\right| n^{\prime}\right\rangle$.

If we neglect $\mathcal{H}_{R}^{(1)}$ as in strict one-dimensional systems, Eq. (8) involves a single mode $n$. If, in addition, the spin axis is chosen along $y$ then the two spin modes uncouple and no spin oscillation is allowed; in other directions $(x$ or $z)$ a rigid spin precession should be expected if all the contribution between parenthesis in Eq. (10) is assumed constant. This precession is the underlying working mechanism of the Datta-Das spin transistor. ${ }^{7}$ Below we investigate the solution of Eq. (8) in the general case in order to analyze the robustness of the spin-precession scenario when $\mathcal{H}_{R}^{(1)}$ is included and when space inhomogeneity in $\alpha(x)$ is also taken into account. The Appendix contains the details of the employed numerical method to compute the transmission $t_{n^{\prime} s^{\prime}, n s}$, i.e., the probability amplitude from a given left incident mode $n s$ to the right mode $n^{\prime} s^{\prime}$. Then, using the scattering approach the linear-response conductance is given by

$$
G=G_{0} \sum_{n s, n^{\prime} s^{\prime}}\left|t_{n^{\prime} s^{\prime}, n s}\right|^{2},
$$

where $G_{0}=e^{2} / h$ is the conductance quantum. For later discussion on the polarization of the transmitted current we also define the polarized conductance $G_{p}$

$$
G_{p}=G_{0} \sum_{n s, n^{\prime} s^{\prime}} s^{\prime}\left|t_{n^{\prime} s^{\prime}, n s}\right|
$$

and the relative polarization $p(-1 \leq p \leq 1)$

$$
p=\frac{G_{p}}{G} .
$$

We shall pay special attention to the multichannel case considering energies $E$ in Eq. (8) such that up to ten propagating modes are active in the leads. The Rashba intensity will be given below in units of $\hbar \omega_{0} \ell_{0}$, i.e., in relative terms with respect to the confinement energy and length. Therefore, the confinement is what determines whether the maximum $\alpha_{0}$ attainable in practice corresponds to the weak or to the strong coupling limit. Of course, this way one can always reach the strong coupling limit $\alpha_{0} \gg \hbar \omega_{0} \ell_{0}$ for a fixed $\alpha_{0}$, provided the confinement is weak enough. For instance, assuming InAs parameters, ${ }^{5,6} \alpha_{0}=10 \mathrm{meV}$ nm corresponds to $\alpha_{0}=2 \hbar \omega_{0} \ell_{0}$ for $\hbar \omega_{0} \approx 0.01 \mathrm{meV}$ and $\ell_{0} \approx 0.5 \mu \mathrm{m}$. This is a wire width which is within the scope of present experimental techniques. ${ }^{48}$

\section{RESULTS FOR SPIN-POLARIZED LEADS}

Figure 2 shows the results for polarized leads oriented along $x$. When $\mathcal{H}_{R}^{(1)}$ is neglected the conductance for five and ten propagating modes displays an almost sinusoidal behavior with only minor distortions. These deviations, which are enhanced in the single-mode case, can be attributed to the quantum interference with the Rashba dot. ${ }^{13}$ The present results confirm, therefore, the precession scenario mentioned above but only when the number of modes is large enough and interband coupling is neglected. Quite remarkably, however, this scenario is not robust with the inclusion of $\mathcal{H}_{R}^{(1)}$. When the full Rashba interaction is considered only for small values of $\alpha_{0}$ the conductance behaves in a regular way. Very rapidly as $\alpha_{0}$ increases $G$ fluctuates in a staggered way that resembles the conductance fluctuations of disordered systems. The mean value, in units of $G_{0}$, is $\approx 0.5 N_{p}$, with $N_{p}$ the number of active channels, while the amplitude of the fluctuation decreases when $N_{p}$ increases. A similar decreasing behavior was obtained in Refs. 19-21 for a vanishing $\omega_{0}$ but without the disordered fluctuations at strong $\alpha_{0}$ 's due to the absence of interband coupling in the purely $2 \mathrm{D}$ geometry.

The existence of the first conductance minimum has been clearly seen in the experiments of Ref. 10. Our results are in agreement with this experiment but they also predict that successive maxima and minima are heavily distorted or even fully washed out. It is also worth noticing that the first conductance minimum for the black dots occurs at a slightly 

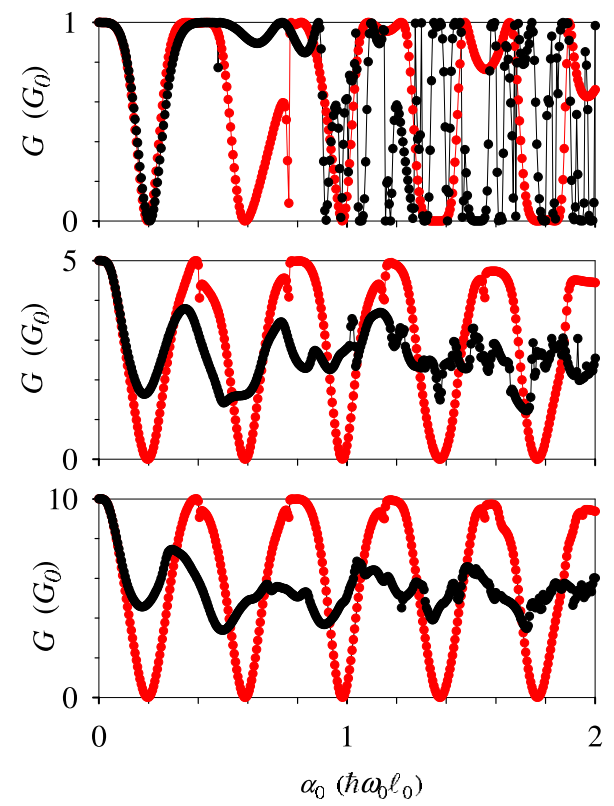

FIG. 2. (Color online) Conductance as a function of Rashba coupling intensity. Black corresponds to the complete Rashba interaction while gray (red color) to the neglect of $\mathcal{H}_{R}^{(1)}$. The leads are spin-polarized along $x$. Upper, intermediate, and lower panel correspond to $N_{p}=1,5$, and 10 propagating modes, respectively. We take the parameters $\ell=8 \ell_{0}, E=N_{p} \hbar \omega_{0}, \Delta_{\ell}=\Delta_{r}=10 \hbar \omega_{0}, d_{\ell}=d_{r}=10 \ell_{0}$, and $a=0.1 \ell_{0}$.

lower value of $\alpha_{0}$ than that of the gray (red color) data, indicating that the minima $\alpha_{\text {min }}$ are somewhat contracted with respect to the simple prediction from the Rashba dot length: $2 m \ell \alpha_{\min }=n \pi \hbar^{2}$, with $n=1,2, \ldots$ (red symbols).

Figure 3 contains the results for polarized leads along $x$ but in antiparallel directions. In this case, when $\alpha_{0} \approx 0$ the conductance vanishes due to the spin-valve effect. As $\alpha_{0}$
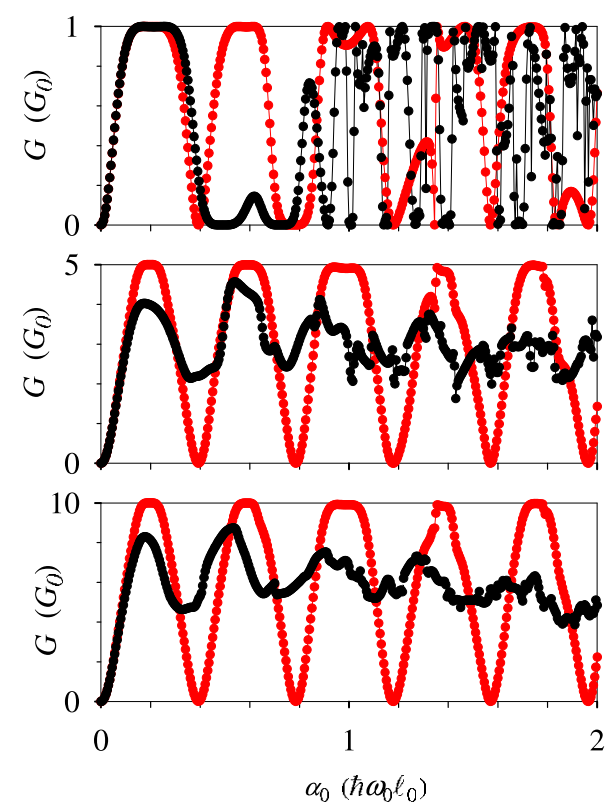

FIG. 3. (Color online) Same as Fig. 2 for polarized leads along $x$ but in antiparallel orientations, i.e., $\Delta_{\ell}=10 \hbar \omega_{0}$ and $\Delta_{r}=-10 \hbar \omega_{0}$.
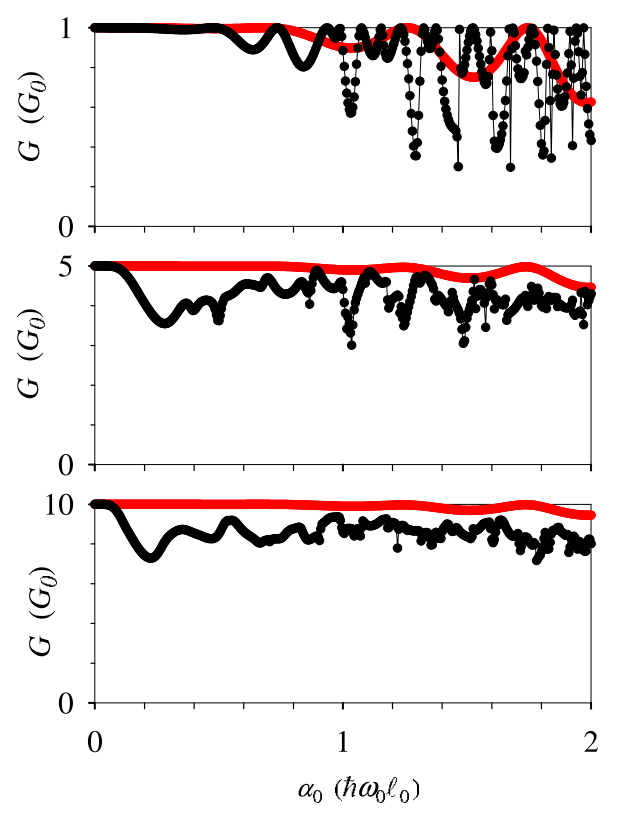

FIG. 4. (Color online) Same as Fig. 2 for parallel polarized leads along $y$.

increases, however, the conductance rises and the spin-valve effect is effectively destroyed by the presence of the Rashba dot. For big enough values the system behaves similarly to the case of parallel polarized leads (Fig. 2), displaying irregular oscillations around a mean value $\approx N_{p} / 2$. For strong spin-orbit couplings and high number of modes no clear distinction between parallel and antiparallel orientations is then to be expected. This is a consequence of the strong subband mixing. In fact, if $\mathcal{H}_{R}^{(1)}$ is neglected (red symbols) there is a full correspondence between the conductance nodes of the parallel geometry with the maxima of the antiparallel one; as could expected from the simplified rigid precession scenario.

The above results are not modified if other values of $\Delta_{\ell, r}$ are used, provided they are large enough to ensure full polarization of the leads. The same is true for distances $d_{\ell, r}$. They should be large enough to allow the decay of evanescent states at the interfaces with the Rashba dot and at the points where Stoner fields are switched on.

We consider next polarized leads along $y$ and $z$; that is, in directions that are perpendicular to the quantum wire. For $z$ polarizations the results are very similar to the $x$ ones already discussed and thus will not be shown. Figures 4 and 5 contain the results for $y$-polarized parallel and antiparallel leads. A first conspicuous difference with the results of Figs. 2 and 3 is that the gray symbols (red color) do not display wide sinusoidal oscillations. The conductance when $\mathcal{H}_{R}^{(1)}$ is neglected is actually maximal for the parallel case and stays rather constant with some small oscillations at large $\alpha$ 's that disappear when the number of channels increases. On the other hand, $G$ vanishes for the antiparallel orientation. We understand this spin-valve behavior as a complete absence of spin precession, resulting from the fact that $\mathcal{H}_{R}$ is spin diagonal in this approximation [cf. Eq. (10)].

Including $\mathcal{H}_{R}^{(1)}$ in the $y$-polarized geometry again yields qualitative modifications of the linear conductance (black symbols in Figs. 4 and 5). Except for the antiparallel one- 

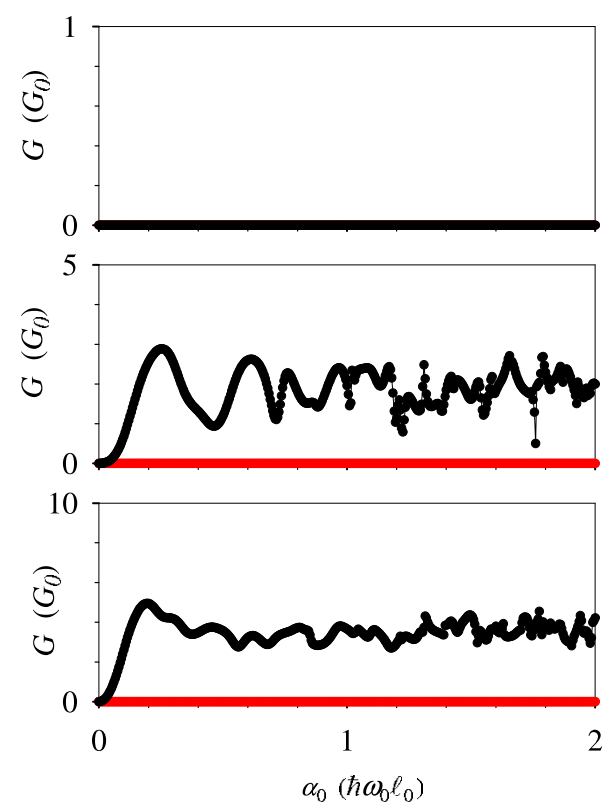

FIG. 5. (Color online) Same as Fig. 2 for polarized leads along $y$ in antiparallel orientation.

channel case, $G$ shows staggering behavior at large $\alpha_{0}$ 's, quite similarly to the $x$-polarized results. On average, the conductance is somewhat reduced from the maximal value in the parallel case (Fig. 4) and, remarkably, takes a finite value in the antiparallel distribution (Fig. 5). For $\alpha_{0} \sim 0.2 \hbar \omega_{0} \ell_{0}$ the antiparallel conductance has already reached a value close to $N_{p} / 2$ and to the eventual saturation value. The Rashba coupling is thus quite effective in allowing transmission by flipping spins of the polarized incoming electrons toward the opposite spin orientation of the outgoing ones. The single channel limit (upper panel of Fig. 5) is obviously an exception since even the black symbols vanish in this case. This is easily understood noticing that the incident $n s=0+$ mode couples in the Rashba dot with modes $1-, 2+, \ldots$, but not with $0-$, which is the only propagating mode in the right lead. Therefore, no conduction is possible under these conditions.

Experimentally, the absence of conductance oscillation in the parallel $y$-oriented configuration has been confirmed. ${ }^{10}$ Our results reproduce that behavior (Fig. 4) and they also suggest the antiparallel $y$ orientation (Fig. 5) as an interesting configuration for a spin-orbit-controlled device. Indeed, the initial rise of conductance in the multichannel case, interpreted above as a Rashba-induced destruction of the spin valve, could be used as the conducting state of the device. One should check, however, that the evolution of $G\left(\alpha_{0}\right)$ from zero to the higher values remains smooth for increasing numbers of propagating channels. The present results do not elucidate this point but they seem to indicate that for $N_{p}=10$ propagating modes the initial rise of $G\left(\alpha_{0}\right)$ occurs more rapidly than for $N_{p}=5$. In a future work we shall treat the continuum case, having an infinite number of transverse states, using a different approach from the present one.

The results shown above are not much modified if the interfaces with the Stoner fields at distances $d_{\ell}$ and $d_{r}$ to the left and right of the Rashba dot, respectively (see Fig. 1), are smoothed by increasing the corresponding Fermi-function parameter. ${ }^{49}$ This confirms that the conductance modifications are an effect of the Rashba dot and not of the Stoner field interfaces. Indeed, the more diffuse the interface, the more reflectionless and thus more ideal is the description of the contact. In the next section we shall discuss the case of nonpolarized leads $\left(\Delta_{0}=0\right)$ but we have also calculated some cases of partial polarization by decreasing $\Delta_{0}$ when both $s$ $=+$ and - transverse states are active although their number is not perfectly balanced. We have found that the conductance is qualitatively similar to the fully polarized case with irregular behavior at large values of $\alpha_{0}$.

\section{RASHBA POLARIZERS}

It has been recently pointed out ${ }^{34,42}$ that a Rashba dot can act as a current polarizer in such a way that when a nonpolarized current enters the dot from the left, the transmitted current to the right may attain an important degree of spin polarization in $y$ direction. For this to occur, it has been shown that at least two propagating modes of opposite spin must interfere. ${ }^{34,42}$ In wires with parabolic transverse confinement this means that the energy should at least exceed $1.5 \hbar \omega_{0}$ such that the four modes $\{0+, 0-, 1+, 1-\}$ are active and the interference occurs in subsets $\{0+, 1-\}$ and $\{0-, 1$ $+\}$. The resulting spin polarization is very sensitive to the energy (see Fig. 3 of Ref. 42) and a large enhancement of the polarization $p$, Eq. (13) is obtained when the energy is such that a Fano-type resonance with a quasibound state from a higher evanescent band is formed. This type of resonances which lead to the Fano-Rashba effect was investigated in Ref. 13. The polarization of the transmitted current is zero if, instead of $y$, other direction for the quantization axis are chosen.

The preference for the transverse $y$ direction in polarization is an example of chirality induced by the Rashba interaction. This is possible even with a time-reversal invariant Hamiltonian such as Eq. (2) because our boundary condition (left incidence) is not time reversal invariant. Indeed, if we consider the time-reversed boundary condition, i.e., incidence from the right, the current transmitted to the left is polarized in the opposite direction. The superposition of both solutions completely restores the symmetry without any preferred spin direction. The reversal of the polarization for the right-to-left transmission can be seen as a peculiar behavior of Rashba polarizers that makes them fragile in the presence of magnetic barriers like those of Sec. III. Indeed, one could naively think that when the Rashba dot acts as a current polarizer the left-to-right transmission with $y$-magnetized leads should be very high in parallel configuration and very low in antiparallel configuration. This is not the case, however, because of multiple backward and forward reflections with their associated inversions of $p$ (see lower panels of Figs. 4 and 5).

In this section we assume nonmagnetic leads by taking $\Delta_{\ell, r}=0$, i.e., vanishing Stoner fields in Fig. 1, and analyze the evolution of the polarization and the conductance when the number of active channels increases. As shown in Fig. 6 upper panel, high polarizations $p$ are obtained for the mini- 


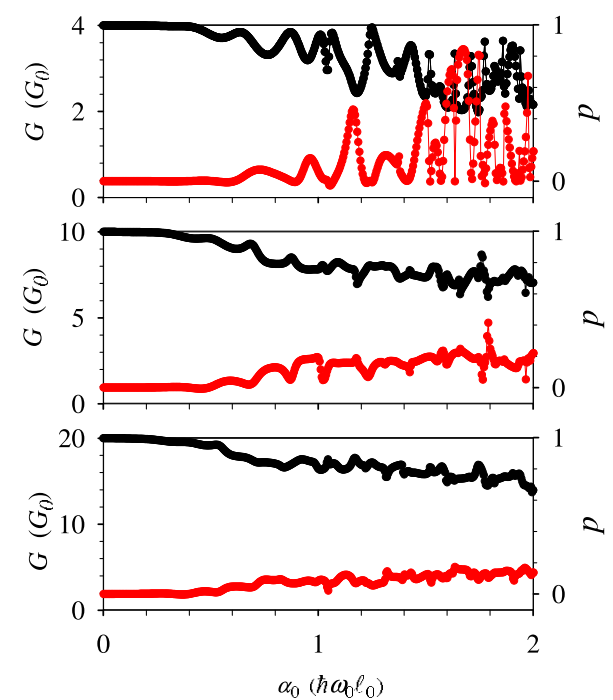

FIG. 6. (Color online) Conductance $G$, black symbols with left scale, and polarization of transmitted current, gray symbols (red in color) with right scale, as a function of the Rashba intensity. We have used the same parameters as in Fig. 2, except for the Stoner fields which are here taken to vanish. Upper, intermediate, and lower panel correspond to $N_{p}=4,10$, and 20 propagating modes, respectively.

mal number of channels $N_{p}=4$ and strong spin-orbit intensities $\alpha_{0}$. The clear correlation between $G$ and $p$, conductance minima correspond to maxima in polarization, indicate that this is an effect connected with the formation of quasibound states that tend to block the current for a given spin direction. When the number of channels is increased (lower panels of Fig. 6) both $G$ and $p$ show reduced staggering oscillations with increasing $\alpha$, as in Figs. 2-5. There is also an overall tendency to smoothly reduce $G$ and increase $p$ in a linear way with $\alpha$. With increasing number of channels the slopes of these straight lines are reduced and for $\alpha_{0} \approx 2 \hbar \omega_{0} \ell_{0}$ the polarization reaches the values $\approx 0.2$ and $\approx 0.1$ for 10 and 20 propagating channels, respectively. In almost all cases the polarization is positive, indicating that the transmitted current is preferentially polarized along $+y$.

\section{Smooth interfaces}

In this section we discuss how the results are affected by the way in which the Rashba field is switched on spatially. For this, we vary the parameter $a$ in the Fermi functions describing the transitions shown in Fig. $1{ }^{49}$ For large values of $a$ the edges are quite smooth and correspond to an adiabatic turn-on or turn-off in space. On the contrary, abrupt changes are given by the limit $a \rightarrow 0$. Our method is based on a grid discretization of the variable $x$ and its only requirement is that the grid should be fine enough to describe the spatial variations.

The results discussed above have been obtained using $a$ $=0.1 \ell_{0}$, a rather small value describing abrupt transitions in space. We have checked that either using a smaller value $a$ $=0.05 \ell_{0}$ or a larger value $a=\ell_{0}$ the behaviors of the conductance in the presence of polarized leads discussed in Sec. II,
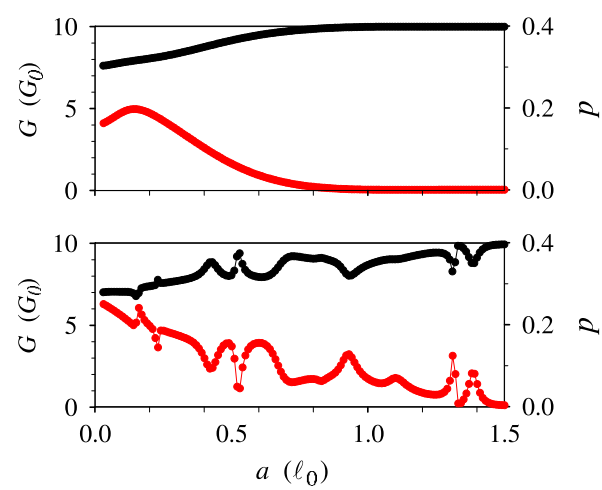

FIG. 7. (Color online) Conductance $G$, black symbols with left scale, and polarization of transmitted current, gray symbols (red in color) with right scale, as a function of the diffusivity $a$ in the Fermi functions describing the spatial transitions in Fig. 1. We have used the same parameters as in Fig. 6, and a value of the Rashba intensity $\alpha_{0}=\hbar \omega_{0} \ell_{0}$ and $2 \hbar \omega_{0} \ell_{0}$ for the upper and lower panels, respectively.

namely, the staggering for high values of $\alpha_{0}$ and the modification due to intersubband coupling, are not qualitatively changed. Of course, it should be fulfilled that the Rashba dot length $\ell$ is much greater than $a$ in order to still allow the transition to reach to the saturation value $\alpha_{0}$. More delicate is the polarization $p$ discussed in the preceding subsection and Fig. 6. In Fig. 7 we show the evolution with $a$ of $G$ and $p$ when $N_{p}=5$ channels are propagating in the wire. The polarization vanishes when $a$ increases, indicating that smooth edges do not favor the appearance of polarized currents. In this diffuse-edge limit the conductance takes the maximal value $G=N_{p} G_{0}$ as in a purely ballistic wire without any Rashba dot. The evolution for $\alpha_{0}=\hbar \omega_{0} \ell_{0}$ (upper panel) is quite smooth but for $\alpha_{0}=2 \hbar \omega_{0} \ell_{0}$ (lower panel) superimposed to the overall behavior we find irregular maxima and minima as in previous results.

\section{CONCLUSIONS}

Recent experiments have proved the feasibility of the spin transistor proposed by Datta and Das ${ }^{7,10}$ some years ago. This device, usually presented as a paradigm of spintronics, is expected to open new ways to overcome present limitations of electronics. In this paper we have discussed some specific aspects related to the Rashba interaction, including the so-called intersubband coupling, relevant for a better understanding of the physical mechanisms behind the spin transistors and spin polarizers.

Taking the wire containing the Rashba dot oriented along $x$ we have analyzed the transmission in the presence of polarized leads along $x, y$, or $z$, and with increasing number of propagating channels. The cases of parallel and antiparallel polarized leads along $x$ and $y$ have been explicitly shown. The evolution with Rashba intensity shows dramatic modifications when the Rashba intersubband coupling is included. These modifications are specially relevant at strong values of $\alpha_{0}$, where staggering oscillations of $G$ have been found. In general, only a first smooth oscillation of $G\left(\alpha_{0}\right)$ remains 
when the full Rashba interaction is considered while successive ones are heavily distorted or even fully washed out. The spin-valve behavior is effectively destroyed by the Rashba dot and the conductance for both parallel and antiparallel leads is relatively high.

The role of Rashba dots as spin polarizers has been discussed and explicitly calculated assuming the leads to be nonpolarized. A smooth linear increase in $p$ with Rashba intensity has been observed in the multichannel case. In the limit of adiabatic transitions the polarization vanishes. These overall smooth behaviors are superimposed by irregular changes for high values of $\alpha_{0}$.

\section{ACKNOWLEDGMENTS}

Useful discussions with M.-S. Choi are gratefully acknowledged. This work was supported by the MICINN (Spain) under Grant No. FIS2008-00781.

\section{APPENDIX: RESOLUTION METHOD}

This appendix gives some details of the practical method to solve Eq. (8) and the corresponding boundary conditions. We use a method based on the quantum transmitting boundary algorithm. ${ }^{50,51}$ A fictitious partitioning of the system in central and asymptotic regions (contacts) is introduced. The boundaries for the left and right contacts are at $x_{\ell}$ and $x_{r}$, respectively. In the contacts the band amplitudes take the form

$$
\psi_{n s}(x)=a_{c, n s} e^{i s_{c} k_{c, n s}\left(x-x_{c}\right)}+b_{c, n s} e^{-i s_{c} k_{c, n s}\left(x-x_{c}\right)},
$$

where $c=\ell, r$ is a label referring to left $(\ell)$ and right $(r)$ contacts, respectively, and we defined $s_{\ell}=1$ and $s_{r}=-1$. The incident and reflected amplitudes for a given mode $n s$ and contact $c$ are given by $a_{c, n s}$ and $b_{c, n s}$, respectively. This expression is for a propagating channel in contact $c$, for which $\varepsilon_{n}+\left|\Delta_{c}\right|+s \Delta_{c}<E$ and its corresponding wave number

$$
k_{c, n s}=\sqrt{2 m^{*}\left(E-\varepsilon_{n}-\left|\Delta_{c}\right|-s \Delta_{c}\right)} / \hbar
$$

is a real number. Equation (A1) also applies to evanescent modes, $\varepsilon_{n}+\left|\Delta_{c}\right|+s \Delta_{c}>E$, if we assume in this case $a_{c, n s}=0$ and a purely imaginary wave number

$$
k_{c, n s}=i \sqrt{2 m^{*}\left(\varepsilon_{n}+\left|\Delta_{c}\right|+s \Delta_{c}-E\right)} / \hbar .
$$

Notice that the output amplitudes can be obtained from the wave function right at the interface

$$
b_{c, n s}=\psi_{n s}\left(x_{c}\right)-a_{c, n s} .
$$

Substituting Eq. (A4) in Eq. (A1) we obtain

$$
\psi_{n s}(x)-\psi_{n s}\left(x_{c}\right) e^{-i s_{c} k_{c, n s}\left(x-x_{c}\right)}=2 i a_{c, n s} \sin \left[s_{c} k_{c, n s}\left(x-x_{c}\right)\right],
$$

that is, the quantum-transmitting-boundary equation for the contacts.

Equations (8) and (A5), for the central and contact regions, respectively, form a closed set that does not invoke the wave function at any external point. Of course, this is not true for any of these two subsets separately since central and contact regions are connected through the derivative in Eq. (8) and of $\psi_{n s}\left(x_{c}\right)$ in Eq. (A5). In practice, we use a uniform grid in $x$ with $n$-point formulas for the derivatives ( $n$ $\approx 5-11$ ) and truncate the expansion in transverse bands, Eq. (4), to include typically 30-60 terms. The resulting sparse linear problem is then solved using routine ME48. ${ }^{52}$
${ }^{1}$ M. N. Baibich, J. M. Broto, A. Fert, F. Nguyen Van Dau, F. Petroff, P. Etienne, G. Creuzet, A. Friederich, and J. Chazelas, Phys. Rev. Lett. 61, 2472 (1988).

${ }^{2}$ G. Binasch, P. Grünberg, F. Saurenbach, and W. Zinn, Phys. Rev. B 39, 4828 (1989).

${ }^{3}$ J. Fabian, A. Matos-Abiague, C. Ertler, P. Stano, and I. Zutic, Acta Phys. Slov. 57, 565 (2007).

${ }^{4}$ E. I. Rashba, Fiz. Tverd. Tela (Leningrad) 2, 1224 (1960) [Sov. Phys. Solid State 2, 1109 (1960)].

${ }^{5}$ J. Nitta, T. Akazaki, H. Takayanagi, and T. Enoki, Phys. Rev. Lett. 78, 1335 (1997).

${ }^{6}$ G. Engels, J. Lange, Th. Schäpers, and H. Lüth, Phys. Rev. B 55, R1958 (1997).

${ }^{7}$ S. Datta and B. Das, Appl. Phys. Lett. 56, 665 (1990).

${ }^{8}$ G. Schmidt, D. Ferrand, L. W. Molenkamp, A. T. Filip, and B. J. van Wees, Phys. Rev. B 62, R4790 (2000).

${ }^{9}$ J. Schliemann, J. C. Egues, and D. Loss, Phys. Rev. Lett. 90, 146801 (2003).

${ }^{10}$ H. C. Koo, J. H. Kwon, J. Eom, J. Chang, S. H. Han, and M. Johnson, Science 325, 1515 (2009).

${ }^{11}$ I. A. Shelykh and N. G. Galkin, Phys. Rev. B 70, 205328 (2004).

${ }^{12}$ L. Zhang, P. Brusheim, and H. Q. Xu, Phys. Rev. B 72, 045347
(2005); J.-S. Jeong and H.-W. Lee, ibid. 74, 195311 (2006).

${ }^{13}$ D. Sánchez and Ll. Serra, Phys. Rev. B 74, 153313 (2006).

${ }^{14}$ R. López, D. Sánchez, and Ll. Serra, Phys. Rev. B 76, 035307 (2007).

${ }^{15}$ U. Fano, Phys. Rev. 124, 1866 (1961).

${ }^{16}$ Ll. Serra, D. Sánchez, and R. López, Phys. Rev. B 72, 235309 (2005).

${ }^{17}$ Yu. V. Pershin, J. A. Nesteroff, and V. Privman, Phys. Rev. B 69, 121306(R) (2004).

${ }^{18}$ D. Sánchez, L1. Serra, and M.-S. Choi, Phys. Rev. B 77, 035315 (2008).

${ }^{19}$ M. G. Pala, M. Governale, J. König, and U. Zülicke, Europhys. Lett. 65, 850 (2004).

${ }^{20}$ A. Zainuddin, S. Hong, L. Siddiqui, and S. Datta, arXiv:1001.1523 (unpublished).

${ }^{21}$ P. Agnihotri and S. Bandyopadhyay, Physica E 42, 1736 (2010).

${ }^{22}$ A. A. Kiselev and K. W. Kim, Appl. Phys. Lett. 78, 775 (2001).

${ }^{23}$ M. Governale, D. Boese, U. Zülicke, and C. Schroll, Phys. Rev. B 65, 140403(R) (2002).

${ }^{24} \mathrm{R}$. Ionicioiu and I. D'Amico, Phys. Rev. B 67, 041307(R) (2003).

${ }^{25}$ G. Usaj and C. A. Balseiro, Phys. Rev. B 70, 041301(R) (2004). 
${ }^{26}$ A. O. Govorov, A. V. Kalameitsev, and J. P. Dulka, Phys. Rev. B 70, 245310 (2004).

${ }^{27}$ M. Khodas, A. Shekhter, and A. M. Finkel'stein, Phys. Rev. Lett. 92, 086602 (2004).

${ }^{28}$ M. Yamamoto, T. Ohtsuki, and B. Kramer, Phys. Rev. B 72, 115321 (2005).

${ }^{29}$ M. Eto, T. Hayashi, and Y. Kurotani, J. Phys. Soc. Jpn. 74, 1934 (2005).

${ }^{30}$ J. I. Ohe, M. Yamamoto, T. Ohtsuki, and J. Nitta, Phys. Rev. B 72, 041308(R) (2005).

${ }^{31}$ P. G. Silvestrov and E. G. Mishchenko, Phys. Rev. B 74, 165301 (2006).

${ }^{32}$ A. W. Cummings, R. Akis, and D. K. Ferry, Appl. Phys. Lett. 89, 172115 (2006).

${ }^{33}$ F. Zhai and H. Q. Xu, Phys. Rev. B 76, 035306 (2007).

${ }^{34}$ C. A. Perroni, D. Bercioux, V. M. Ramaglia, and V. Cataudella, J. Phys.: Condens. Matter 19, 186227 (2007).

${ }^{35}$ J.-F. Liu, Z.-C. Zhong, L. Chen, D. P. Li, C. Zhang, and Z. S. Ma, Phys. Rev. B 76, 195304 (2007).

${ }^{36}$ H.-F. Lü and Y. Guo, Phys. Rev. B 76, 045120 (2007); Appl. Phys. Lett. 91, 092128 (2007).

${ }^{37}$ M. Scheid, A. Pfund, D. Bercioux, and K. Richter, Phys. Rev. B 76, 195303 (2007).

${ }^{38}$ V. M. Apel, P. A. Orellana, and M. Pacheco, Nanotechnology 19, 355202 (2008).

${ }^{39}$ A. Aharony, O. Entin-Wohlman, Y. Tokura, and S. Katsumoto, Phys. Rev. B 78, 125328 (2008).

${ }^{40}$ F. Zhai, K. Chang, and H. Q. Xu, Appl. Phys. Lett. 92, 102111
(2008).

${ }^{41}$ P. Debray, J. Wan, S. M. S. Rahman, R. S. Newrock, M. Cahay, A. T. Ngo, S. E. Ulloa, S. T. Herbert, M. Muhammad, and M. Johnson, Nat. Nanotechnol. 4, 759 (2009).

${ }^{42}$ M. M. Gelabert, D. Sánchez, R. López, and L1. Serra, Phys. Status Solidi C 6, 2123 (2009).

${ }^{43}$ O. Entin-Wohlman, A. Aharony, Y. Tokura, and Y. Avishai, Phys. Rev. B 81, 075439 (2010).

${ }^{44}$ E. N. Bulgakov and A. F. Sadreev, Phys. Rev. B 66, 075331 (2002).

${ }^{45}$ F. Zhai and H. Q. Xu, Phys. Rev. Lett. 94, 246601 (2005).

${ }^{46}$ A. A. Kiselev and K. W. Kim, Phys. Rev. B 71, 153315 (2005).

${ }^{47}$ A. Auerbach, Interacting Electrons and Quantum Magnetism (Springer-Verlag, New York, 1994).

${ }^{48}$ Th. Schäpers, J. Knobbe, and V. A. Guzenko, Phys. Rev. B 69, 235323 (2004).

${ }^{49}$ The inhomogeneous Rashba intensity is given by $\alpha(x)$ $=\alpha_{0}[F(x, \ell / 2)-F(x,-\ell / 2)]$ where the Fermi functions are $F\left(x, x_{0}\right)=\left\{1+\exp \left[\left(x-x_{0}\right) / a\right]\right\}^{-1}$ and $a$ is a parameter modeling the diffusivity of the interface. Similar expressions for the gate potential $V_{g}(x)$ and Stoner field $\Delta(x)$ are used.

${ }^{50}$ C. S. Lent and D. J. Kirkner, J. Appl. Phys. 67, 6353 (1990).

${ }^{51}$ Progress in Industrial Mathematics 2006, edited by L. L. Bonilla, M. Moscoso, G. Platero, and J. M. Vega (Springer, Berlin, 2008), p. 449.

${ }^{52}$ HSL, a collection of FORTRAN codes for large-scale scientific computation. See http://www.hsl.rl.ac.uk (2007). 